\title{
NEUTROPHILIC DERMATOSIS OF THE DORSAL HANDS ASSOCIATED WITH A "MYELOPROLIFERATIVE NEOPLASM, UNCLASSIFIABLE" AND A SIMULTANEOUS CANCER OF COLON
}

\author{
Francisco José Fernández - Fernández ${ }^{1}$, Juan Carlos Álvarez - Fernández², Esperanza Romero - Picos ${ }^{3}$, \\ Juan Antonio Garrido ${ }^{1}$, Pascual Sesma ${ }^{1}$
}

Hospital Arquitecto Marcide, Ferrol, Spain: Department of Internal Medicine ${ }^{1}$, Department of Pathology ${ }^{2}$, Department of Haematology $y^{3}$

Summary: Neutrophilic dermatosis of the dorsal hands is a variant of Sweet's syndrome. We herein describe an 83-yearold woman with a neutrophilic dermatosis of the dorsal hands associated with a "myeloproliferative neoplasm, unclassifiable" and a simultaneous cancer of colon. To our knowledge, and after a search in PubMed, the association of Sweet's syndrome with a "myeloproliferative neoplasm, unclassifiable" and a simultaneous cancer of colon has not previously been reported.

Key words: Sweet's syndrome; Myeloproliferative neoplasms; Colorectal neoplasms

\section{Introduction}

Sweet's syndrome is an entity characterized by a constellation of symptoms, physical features, and pathological findings which include fever, leukocytosis with neutrophilia, tender erythematous to violaceous skin lesions, and a diffuse infiltrate consisting predominantly of mature neutrophils typically located in the upper dermis. Neutrophilic dermatosis of the dorsal hands is a variant of this syndrome with a morphology and response to treatment of the lesions similar to those of Sweet's syndrome, but with the lesions mainly located on the dorsal part of the hands. Sweet's syndrome is associated with a variety of underlying conditions in as many as 50 percent of patients (5) and this dermatosis may occur as a paraneoplastic syndrome. The most common underlying neoplasm is acute myelogenous leukaemia. Among solid tumours, carcinomas of the genitourinary organs, breast, and gastrointestinal tract, are the most common (2). We present a case of neutrophilic dermatosis of the dorsal hands in a patient with a "myeloproliferative neoplasm, unclassifiable" and a simultaneous cancer of colon.

\section{Case report}

In November, 2007, an 83-year-old woman was admitted to the hospital for evaluation of haematochezia. In her medical history, with the exception of hypertension that was treated with valsartan, no prior pathological conditions were reported. She did not refer anorexia, weight loss or gastrointestinal bleeding previous to her current hospitalization. On physical examination the patient was obese and in mild discomfort. The temperature was $37,1{ }^{\circ} \mathrm{C}$ and the blood pressure was $143 / 67 \mathrm{~mm} \mathrm{Hg}$. The patient's initial laboratory assessment revealed a white-cell count (WBC) of $68,34 \times 10^{9} / \mathrm{L}$ with 89 percent polymorphonuclear cells. The haemoglobin level was $11,7 \mathrm{~g} / \mathrm{dl}$ and the erythrocyte sedimentation rate $40 \mathrm{~mm}$ per hour. Lactate dehydrogenase was high at $795 \mathrm{IU} / \mathrm{L}$ (range 225-450). On the third hospital day, severely painful purulent nodules appeared on the dorsum of her hands and fingers (Fig. 1, on top). These lesions increased in size and number, and developed into lesions with bullous appearance and ulceration (Fig. 1, down). Cultures of cutaneous lesions were negative. A lesional skin biopsy showed an intense neutrophilic infiltration in the dermis with leukocytoclastic debris. The epidermis was spared (Fig. 2). A diagnosis of neutrophilic dermatosis of the dorsal hands was made. Methylprednisolone was administered intravenously, followed by a tapering oral dose of prednisone. The cutaneous lesions began to clear in spite of a rise of the neutrophils induced by glucocorticoids. In the evaluation of her neutrophilic leukocytosis, a haematological profile on the third hospital day showed a WBC of $57,2 \times 10^{9} / \mathrm{L}$, haemoglobin of $9,8 \mathrm{~g} / \mathrm{dL}$ and platelets of $594 \times 10^{9} / \mathrm{L}$. The differential count was $75 \%$ neutrophils, $6 \%$ bands, $3 \%$ metamyelocytes, $1 \%$ myelocytes, $3 \%$ eosinophils, $10 \%$ lymphocytes and $2 \%$ monocytes. The neutrophil alkaline phosphatase (NAP) score was 33 (control 20-40). Philadelphia chromosome on conventional cytogenetic eva- 


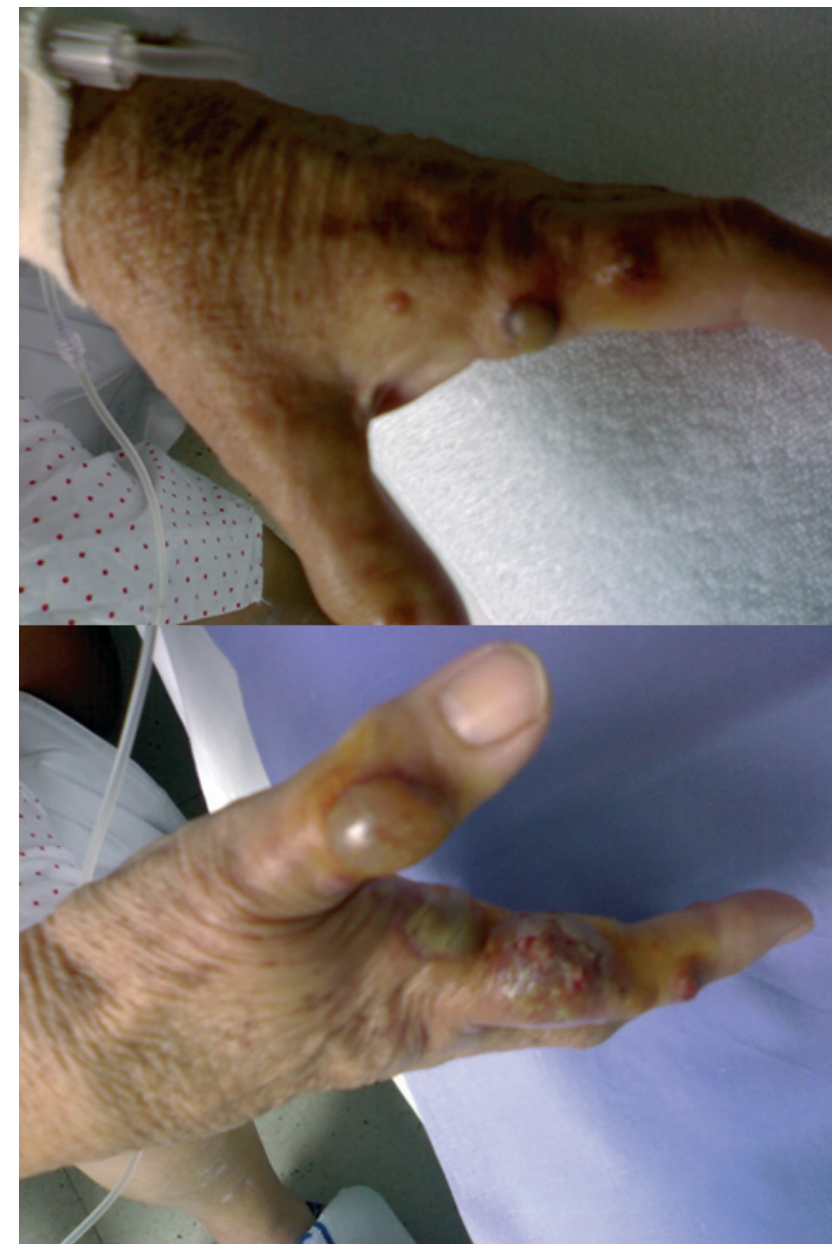

Fig. 1: Purulent nodules on the dorsum of the left hand and index finger (on top). Bullous and ulcerated changes on the thumb and index fingers of the right hand. Yellowing of the skin is due to povidone (down).

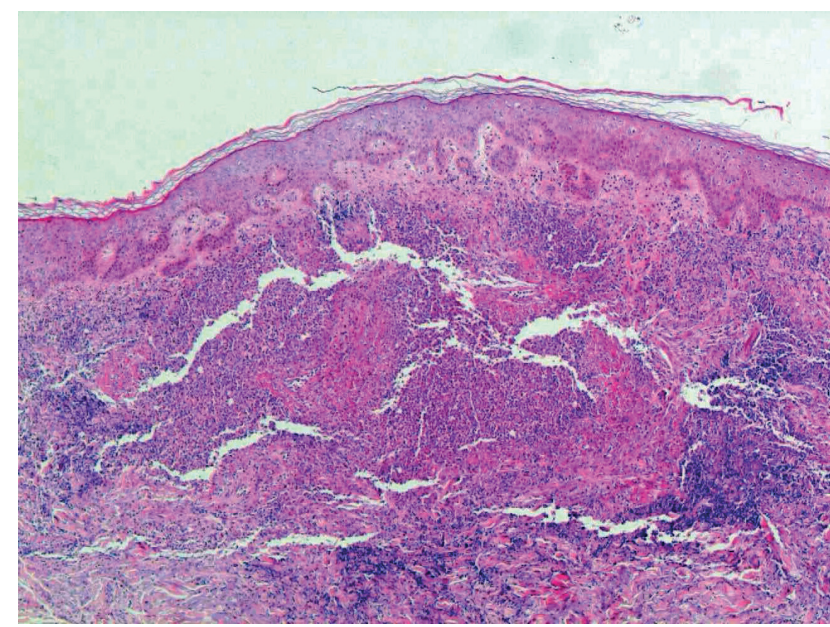

Fig. 2: Skin biopsy specimen showing extensive dermal neutrophilic infiltrate. The epidermis is spared (hematoxylineosin, magnification $\mathrm{x} 40$ ). luation and traditional FISH for BCR-ABL fusion gene were negative. The reverse-transcription polymerase chain reaction for expression of both the p210 and p190 BCR$\mathrm{ABL}$ transcripts on the peripheral blood was negative. Likewise, mutations of JAK 2, PDGFRA and PDGFRB were absent. The trephine bone marrow biopsy showed a marked hypercellular marrow with findings suggestive of a myeloproliferative neoplasm (Fig. 3, on top). There was no evidence of fibrosis or, metastatic infiltration of the bone marrow by the colonic tumour. The bone marrow aspirate showed myeloid hyperplasia without dysplasia (Fig. 3, down). On the other hand, a colonoscopy revealed an adenocarcinoma arising from a villous adenoma in the sigmoid colon, as the source of the lower gastrointestinal bleeding. A computed tomography scan of the chest, abdomen and pelvis demonstrated the presence of splenomegaly, without other abnormalities of interest. The colonic tumour was completely removed by surgery, and histological investigation of the excised portion confirmed the

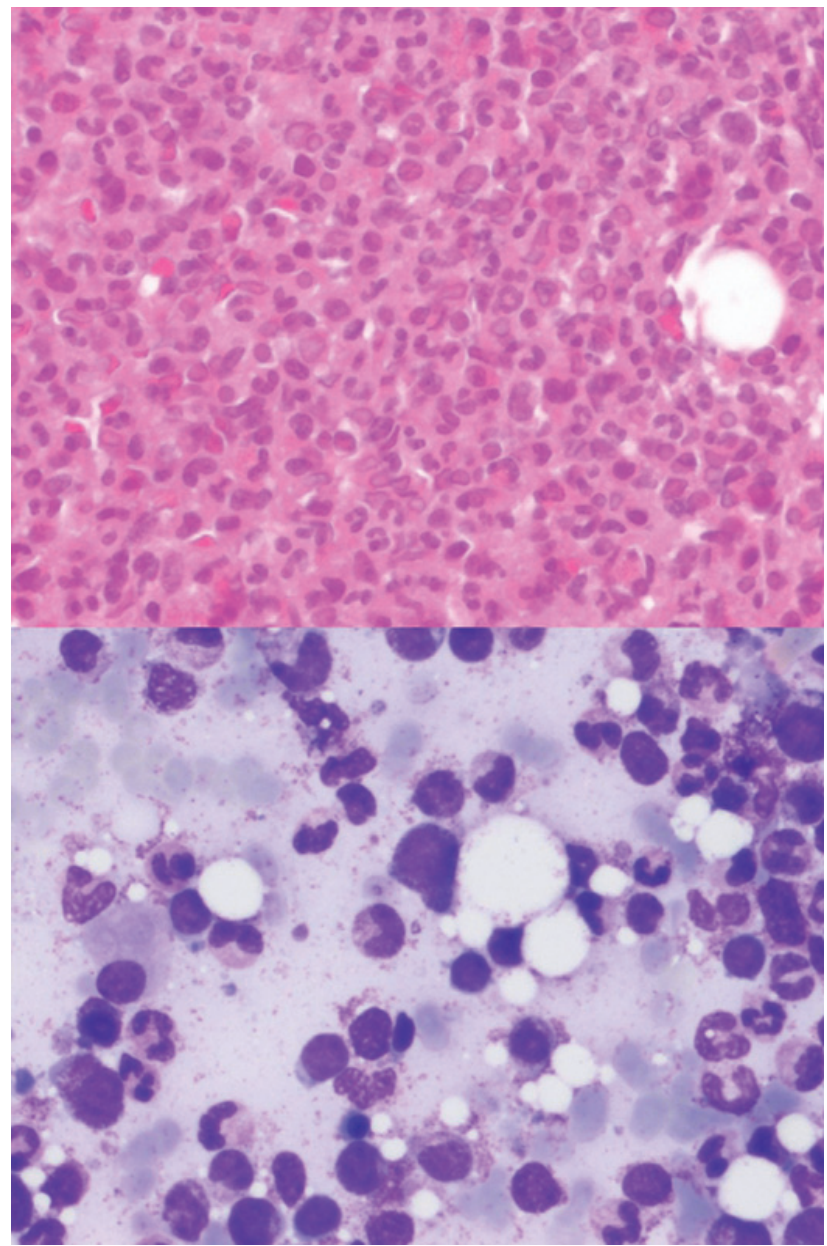

Fig. 3: Bone marrow biopsy showing hypercellular bone marrow for age with myeloid hyperplasia (on top). Bone marrow aspiration specimen. There is no evidence of myelodysplastic features (down). 
existence of a well differentiated adenocarcinoma invading the adipose perivisceral tissue with metastatic involvement in two of the 7 nodes removed. After surgery, haematological abnormalities persisted and the NAP score decreased markedly to 3 (range 20-40), persisting markedly decreased in the follow-up studies. Likewise, there was no monocytosis in peripheral blood studies over a longer span of time. In imaging studies at 2 and 6 months of the colonic tumour resection there was no evidence of metastatic disease of her cancer of colon, presenting only splenomegaly. These alterations after surgery allowed us to exclude that the peripheral blood neutrophilia was reactive to the cancer of colon. In November 2007, an initial diagnosis of Ph-negative chronic myeloid leukaemia was made. According to the 2008 World Health Organization classification system for haematological malignancies, and based on the clinical, molecular and pathological findings, we believe that our patient had a "myeloproliferative neoplasm, unclassifiable". Treatment with hydroxyurea was used to control her symptoms and WBC count. She died about a year after her first presentation, probably by a pulmonary embolism, and there was no evidence of an accelerated phase or blastic crisis in her last revision. Necropsy was not performed.

\section{Discussion}

Sweet's syndrome (the eponym for acute febrile neutrophilic dermatosis) was described by Robert D. Sweet in 1964 (10). Since 1995, several authors have reported a variant of this syndrome with a morphology and response to treatment of the lesions similar to those of Sweet's syndrome, but with the lesions mainly located on the dorsal part of the hands. This variant was named "pustular vasculitis of the dorsal hands" or "neutrophilic dermatosis of the dorsal hands" $(4,9)$. Sweet's syndrome can be idiopathic or associated, among other disorders, with myeloproliferative processes, solid tumours, autoimmune diseases, infections and drugs. Cohen et al. (2) noted that approximately 10-20 percent of the patients with Sweet's syndrome had either a haematological malignancy or a solid malignant tumour. Most of the cancers were hematopoietic, especially acute myelogenous leukaemia and myelodysplastic syndromes, but about 15 percent were due to solid tumours. Furthermore, the drug-induced variant of the dermatosis has been observed in patients following the administration of granulocyte-colony stimulating factor (G-CSF). The pathogenesis of Sweet's syndrome is poorly understood. Currently, it is thought that cytokine dysregulation accounts for most of the clinical, pathological, and laboratory changes of this syndrome. Among the mainly involved cytokines are interleukin (IL)-1, IL-6, IL-8, granulocyte colony-stimulating factor (G-CSF) and granulocyte-macrophage colony stimulating factor (GM-CSF). Several observations have shown that interleukin-1 (IL-1) may be an important mediator in Sweet's syndrome. Thus, acute myelogenous leukaemia is the malignancy most commonly associated with Sweet's syndrome and this syndrome can occur after G-CSF treatment. IL-1 is spontaneously produced by acute myeloblastic leukaemia (AML) cells (8), and this cytokine induces synthesis of GM-CSF and G-CSF (7). Likewise, polymorphonuclear leukocytes may induce IL-8 gene expression in response to GM-CSF, and IL-8 is a potent chemotactic factor for polymorphonuclear leukocytes (6), amplifying the inflammatory response by recruiting additional polymorphonuclear leukocytes into inflammatory sites. In agreement with this pathogenic mechanism, a recent case report showed that anakinra, an IL-1 receptor antagonist, was effective in a patient with refractory Sweet's syndrome (3).

In our patient, in spite of a rise of the neutrophils induced by glucocorticoids, the skin lesions resolved quickly after glucocorticoids introduction. It is known that glucocorticoids inhibit the synthesis of almost all known cytokines (1), and this is primarily achieved through their action on the function of transcription factors, such as nuclear factor kappa B (NF-kB) and activator protein-1 (AP-1) that are required for transcription of proinflammatory mediators. The association of Sweet's syndrome with two simultaneous tumours is not frequent. Thus, in a review of the literature Cohen et al. (2) reported only four patients with solid tumours who also had a haematological malignancy. To our knowledge, and after a search in PubMed, the association of Sweet's syndrome with a "myeloproliferative neoplasm, unclassifiable" and a simultaneous cancer of colon has not previously been reported. In both idiopathic and malignancy-associated Sweet's syndrome there are usually a good response to corticosteroid therapy. In our patient, the myeloproliferative neoplasm and cancer of colon were simultaneously diagnosed, and regression of the skin lesions was obtained with glucocorticoids before the specific treatment of the tumours.

\section{Acknowledgements}

We thank Drs. T. González and C. Quinteiro from the Molecular Medicine Unit - Fundación Pública Galega de Medicina Xenómica. Hospital Clínico Universitario de Santiago de Compostela for help with the molecular analysis of the patient.

\section{References}

1. Auphan N, DiDonato JA, Rosette C, Helmberg A, Karin M. Immunosuppression by glucocorticoids: inhibition of NF-Kappa B activity through induction of I Kappa B synthesis. Science 1995;270:286-90.

2. Cohen PR, Holder WR, Tucker SB, Kono S, Kurzrock R. Sweet syndrome in patients with solid tumors. Cancer 1993;72:2723-31.

3. Delluc A, Limal N, Puéchal X, Frances C, Piette JC, Cacoub P. Efficacy of anakinra, an IL1 receptor antagonist, in refractory Sweet syndrome. Ann Rheum Dis 2008;67:278-9

4. DiCaudo DJ, Connolly SM. Neutrophilic dermatosis (pustular vasculitis) of the dorsal hands: a report of 7 cases and review of the literature. Arch Dermatol $2002 ; 138: 361-5$

5. Kemmett D, Hunter JA. Sweet's syndrome: A clinicopathologic review of twentynine cases. J Am Acad Dermatol 1990;23:503-7. 
6. McCain RW, Dessypris EN, Christman JW. Granulocyte/macrophage colony-stimulating factor stimulates human polymorphonuclear leukocytes to produce interleukin-8 in vitro. Am J Respir Cell Mol Biol 1993:8:28-34.

7. Ogawa Y, Yonekura S, Nagao T. Granulocyte colony-stimulating factor produc tion by human bone marrow fibroblasts stimulated with interleukins. $\mathrm{Am}$ Hematol 1996;52:71-6.

8. Rambaldi A, Torcia M, Bettoni S, et al. Modulation of cell proliferation and cytokine production in acute myeloblastic leukemia by interleukin-1 receptor antagonist and lack of its expression by leukemic cells. Blood 1991;78: $3248-53$

9. Strutton G, Weedon D, Robertson I. Pustular vasculitis of the hands. J Am Acad Dermatol 1995;32:192-8.

10. Sweet RD. An acute febrile neutrophilic dermatosis. Br J Dermatol 1964 76:349-56.

Received: $17 / 02 / 2010$.

Accepted in revised form: 18/06/2010

\section{Corresponding author:}

Dr. F. J. Fernández - Fernández, Department of Internal Medicine, Hospital Arquitecto Marcide, 15405 Ferrol, Spain; e-mail: fjf-fernandez@terra.es 